\title{
Ansiedad de separación: delimitación conceptual, manifestaciones clínicas y estrategias de intervención
}

\author{
C. Villanueva Suárez $z^{\mathrm{a}}$, LJ. Sanz Rodríguez \\ 'Psicóloga clínica. Coordinadora del Programa Infanto-Juvenil. Servicio de Salud Mental. Parla, Madrid. España.

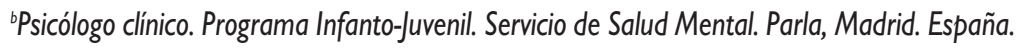

Rev Pediatr Aten Primaria. 2009;1 1:457-69

Carmen Villanueva Suárez, cvillanueva.scsm@salud.madrid.org

\section{Resumen}

En este trabajo queremos destacar la importancia de la intervención en Atención Primaria con los padres y sus bebés en la organización de las interacciones precoces que darán lugar a un apego seguro, base para un buen desarrollo. Para ello, se comentan las intervenciones posibles a propósito de varios casos clínicos.

Palabras clave: Ansiedad de separación, Apego, Consulta terapéutica.

Abstract

In this project we would like to explain the importance of the actions that can be taken in primary care with parents and their babies, to show how early relations will encourage a secure attachment necessary for a good development. With this target, we comment different interventions about several clinical cases.

Key words: Separation anxiety, Attachment, Therapeutic intervention.

\section{Introducción}

Gran parte de los síntomas de los niños que llegan a nuestras consultas están relacionados con las dificultades de autonomía y separación. La ansiedad de separación hace referencia a un estadio del desarrollo infantil en el que los niños sienten angustia cuando se separan de su cuidador principal (generalmente la madre). Esta fase suele comenzar aproximadamente a los 8 meses de edad y durar hasta los 14 meses'.

Antes de los 8 meses, el mundo es tan nuevo para los niños, que apenas son ca-

Los autores declaran no presentar conflictos de intereses en relación con la preparación y publicación de este artículo. 
paces de diferenciar lo que es peligroso de lo que no lo es, de manera que las nuevas situaciones, experiencias o personas no les asustan. Durante el desarrollo normal, esta etapa inicial les permite establecer una familiaridad con sus ambientes. Tras esta fase, la falta de una figura afectiva suele producir temor debido a que el niño es ya capaz de darse cuenta de que sucede algo inusual. Los niños reconocen a sus padres como las personas con quienes se sienten seguros. Cuando se separan de sus padres, sobre todo lejos de casa, se sienten inseguros y amenazados $^{2}$.

Entre los factores que determinan cuál es el cuidador situado en primer lugar son: cantidad de tiempo que el niño pasa con la persona que le cuida; la inversión emocional del adulto respecto al niño; y la frecuencia con la que la persona reaparece ${ }^{3}$.

Así pues, la ansiedad de separación es una fase normal del desarrollo humano. Durante este período el niño puede recurrir a objetos o fenómenos (denominados como objetos transicionales) que están investidos por las características de sus cuidadores principales (como una manta, un peluche o las canciones que tararea el niño para dormirse) y que hacen más tolerable esta ansiedad de separación $n^{4}$. Suele terminar hacia los 14 me- ses de edad, cuando los niños empiezan a entender que los padres siguen existiendo aunque ellos no puedan verlos y que van a volver más tarde. Por tanto, a esta edad es cuando los niños empiezan a querer ser más autónomos y explorar el ambiente que los rodea con más libertad. Son capaces de separarse de sus padres y estar varias horas lejos de ellos, porque han aprendido que los padres siguen estando ahí y volverán a verlos pronto. Ya han desarrollado una sensación de seguridad en sus cuidadores y su ambiente y son capaces de confiar en otras personas además de sus padres ${ }^{5}$.

Pueden distinguirse diversos factores asociados a la aparición de dificultades en el manejo de la ansiedad por separación ${ }^{6,7}$ :

- No haber sido expuesto a una separación paulatina de los padres, quizás por un exceso de sobreprotección de estos y por vivir encerrado en el círculo familiar. Las experiencias repetidas y no traumáticas de separación facilitan el desarrollo de una identidad autónoma e independiente en el niño. La sobreprotección puede darse por diversos motivos: temor de los padres a perder a un hijo que ha sufrido una enfermedad grave, ser hijo único y muy deseado de unos padres mayores, em- 
barazo difícil, pérdida previa de un hijo, problemas previos de esterilidad, insatisfacción marital, ansiedad por separación en algún progenitor, padre o madre muy ansiosos.

- Experiencias traumáticas de separación (vulnerabilidad biológica en el bebé, hospitalización, muerte de uno de los padres, divorcio o separación de estos) en las que se dieran una o más condiciones de las que potencian la ansiedad.

- Refuerzo negativo por parte de los padres a las conductas de apego excesivo del niño. Cuando los padres tienen dificultades para separarse de sus hijos, refuerzan la excesiva dependencia en los niños porque evitan el malestar asociado a la separación y el manejo del mismo ${ }^{8}$.

\section{Organización del apego y ansiedad de separación}

Algunos de los primeros trabajos acerca de los efectos de la separación del niño y sus cuidadores fueron desarrollados por John Bowlby (1907-1990) y le ayudaron a elaborar su teoría del apego. Entendemos por apego al lazo afectivo que se establece entre el niño y una figura específica, que une a ambos en el espacio, perdura en el tiempo, se expresa en la tendencia estable a mantener la proximi- dad y cuya vertiente subjetiva es la sensación de seguridad. Bowlby estableció que la finalidad del sistema de apego es mantener al cuidador accesible y receptivo, lo que resumió en una palabra: disponibilidad. Las conductas de apego que utilizan los bebés son sonreír, vocalizar, llorar para llamar la atención del cuidador y sentir su cercanía.

El trabajo de Bowlby sobre la teoría del apego comenzó cuando, a sus 21 años, trabajó en un hogar de chicos inadaptados y estudió los efectos de la separación del niño de sus padres ${ }^{9}$. En su investigación concluyó que existe una necesidad instintiva del niño a apegarse al cuidador, y considera que los vínculos afectivos son algo biológicamente primario. De este modo, expuso que las relaciones primarias perturbadas madre-hijo, constituyen un precursor clave del trastorno mental ${ }^{10}$.

Bowlby distinguió cuatro períodos en el desarrollo de la vinculación a lo largo de la infancia:

1. Del nacimiento a los 3 meses: dependencia total de la madre y satisfacción de sus necesidades. Etapa de aseguramiento y proximidad, a través del olor y la voz.

2. De los 3 a los 6 meses: el bebe emite señales a otras personas que distingue, sonrisa. 
3. De los 6 meses a los 3 años: intenta mantener la proximidad con las figuras vinculares a las que reconoce perfectamente.

4. A partir de los 3 años ya no tiene necesidad de presencia física de la madre para sentirse seguro.

Partiendo de los trabajos pioneros de Bowlby, a finales de los años setenta, Mary Ainsworth (1913-1999) elaboró un instrumento denominado "situación extraña" con el objetivo de evaluar la manera en que los niños utilizaban a sus figuras de apego como fuente de seguridad, la forma en que reaccionaban ante la presencia de extraños, y en los mo- mentos de separación y reunión con la madre (figura 1).

Ainsworth encontró tres patrones principales de apego (tabla I): el seguro (la categoría más común, que representaba al $65 \%$ de la muestra) y dos formas de apego inseguro: el evitativo (20\% de la muestra) y el ambivalente o resistente $(12 \%)$.

Los bebés con un apego seguro lloran y protestan cuando la madre se va, y la reciben felices cuando regresa. La utilizan como una base segura, al tiempo que se alejan de ella y exploran; pero ocasionalmente, y por seguridad, regresan. Son usualmente cooperativos y

Figura 1. Situación extraña.

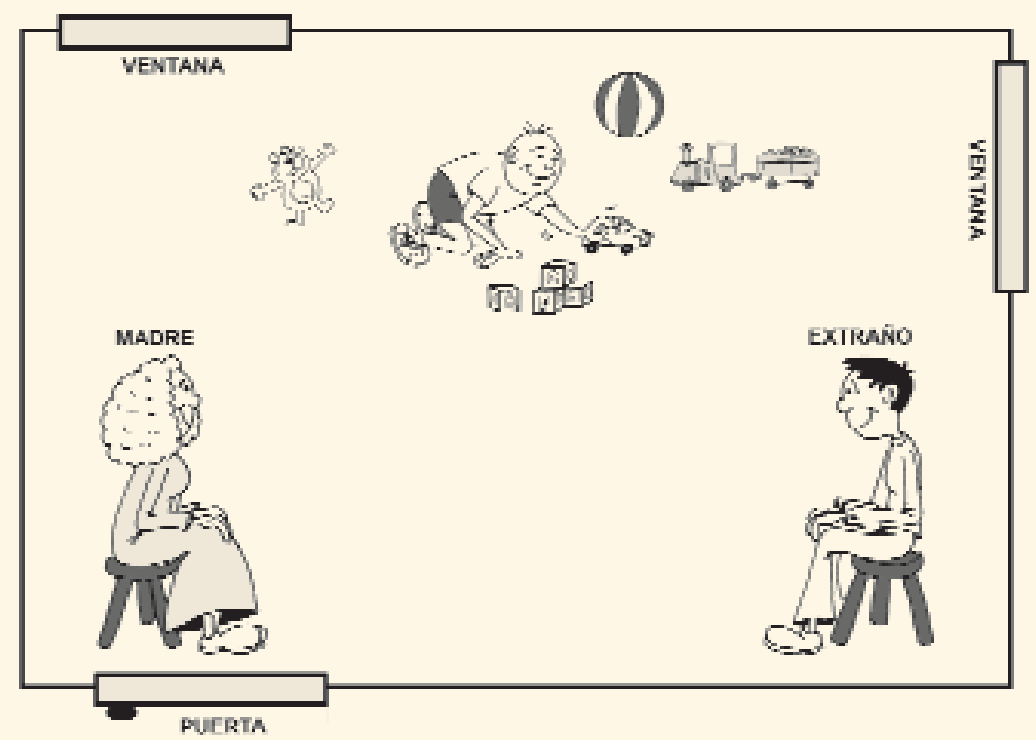


Tabla I. Clasificación del apego en la situación extraña (M. Ainsworth)

\begin{tabular}{lccc}
\hline Apego seguro (65\%) & \multicolumn{3}{c}{ Apego inseguro (35\%) } \\
\cline { 2 - 4 } & $\begin{array}{c}\text { Apego ambivalente/ } \\
\text { resistente (10-12\%) }\end{array}$ & $\begin{array}{c}\text { Apego evitativo } \\
\mathbf{( 2 0 \% )}\end{array}$ & $\begin{array}{c}\text { Apego } \\
\text { desorganizado/ } \\
\text { desorientado (3-5\%) }\end{array}$ \\
\hline - Exploran de forma & - Exploran muy poco & - Poco malestar & - Combinación \\
activa mientras & y se mantienen & en la separación. & de patrón resistente \\
están solos & cerca de la madre. & - Ignoran a la madre & y evitativo; el niño, \\
con la madre. & - Se inquietan mucho & a su regreso. & después de haber \\
- Pueden & en la separación. & - Pueden ser muy & sido separado \\
intranquilizarse & - Ambivalencia & sociables & de la figura de \\
en la separación. & a su regreso & con extraños. & apego, presenta \\
- Contacto físico & (se mantienen & - Muestran una & conductas \\
afectuoso a su & cerca pero se & conducta & contradictorias \\
regreso que & resisten al contacto & exploratoria activa. & a su regreso. \\
les tranquiliza. & físico). & & \\
\hline
\end{tabular}

se enfadan relativamente poco. Los bebés con apego evitativo casi nunca lloran cuando la madre se va y la evitan cuando regresa. Tienden a mostrarse enojados y no la buscan cuando la necesitan. Los bebés con un apego ambivalente (resistente) se sienten ansiosos aun antes de que la madre se vaya y se muestran muy molestos cuando lo hace. Cuando regresa, demuestran su ambivalencia al buscar el contacto con ella; al mismo tiempo, se resisten por medios de pataleos o retorciéndose. Los bebés ambivalentes exploran poco y son difíciles de tranquilizar. Estos tres patrones de apego son universales en todas la culturas en los que han sido estudiados (culturas tan diversas como las de África, China e Israel), aunque el porcentaje de bebés en cada categoría es distinta.

Investigaciones posteriores han identificado un cuarto patrón, el apego desorganizado-desorientado ${ }^{11}$. Los bebés con el patrón desorganizado suelen manifestar conductas inconsistentes y contradictorias. Por ejemplo, pueden recibir a la madre alegremente cuando regresa, pero después se acercan o se alejan sin mirarla; parecen estar confundidos y temerosos. Este podría ser el patrón menos seguro. Es más probable que ocurra en bebés cuyas madres son insensibles, intrusivas o abusivas (tabla I).

El elemento más importante para estudiar la calidad del apego en la situación extraña es el momento de reencuentro entre el cuidador y el niño tras la separa- 
ción. La ansiedad de separación se produce por las reacciones de la madre ante el reencuentro, si es segura, ansiosa o desorganizada. La figura de apego es base fundamental para desarrollar sentimiento de confianza en sí mismo y en el mundo exterior. Es fuente de información emotiva y cognitiva, y fuente de satisfacción de necesidades que ayudarán al niño a regularse y a sentirse seguro con los otros y en el mundo.

La seguridad de apego del niño tendrá consecuencias a largo plazo en sus relaciones personales posteriores y en el desarrollo cognitivo, ya que la relación continua con el cuidador permite entrar en la mentalización, es decir, la representación interna de las relaciones.

\section{Casos clínicos}

A continuación, se describen algunos casos que presentan dificultades relacionadas con la ansiedad de separación. En ellos se ha trabajado fundamentalmente con los padres, sus ansiedades y miedos puestos en las relaciones con sus hijos, impidiendo el proceso de autonomía y separación de los mismos.

Cuando los padres están ansiosos o nerviosos no libidinizan bien y transmiten a los niños unos cuidados desorganizados y un déficit en la regulación de los sistemas biológicos, que termina por ge- nerar los trastornos funcionales: dificultades para dormir, calmarse, comer, etc.

\section{Paloma}

El centro escolar llama a los padres, porque la niña vomita y come muy poco. Paloma tiene 5 años y su madre no sabe como tratarla: "Lo único que hago es regañarla para que no vomite". Cuando no le gusta algo vomita, dice que le duele la tripa, suele vomitar después de desayunar, casi siempre cuando la llevan al colegio. Por las noches sigue tomando biberón, la madre se lo da dormida porque si no, "¡no hay manera!". Con los abuelos la niña come fenomenal.

Los padres refieren que el embarazo y parto trascurrieron sin dificultades. Sin embargo, la crianza resultó complicada porque "era una niña muy difícil, dormía fatal en nuestra habitación y meciéndola...". Recibió lactancia materna hasta los 9 meses, cuando intentó incorporar sólidos, como se atragantaba, se lo daban todo en purés, hasta los 3 años. Consiguieron que se durmiera sola a los 2 años, pero cuando se despertaba, se venía a su cama, porque " $\mathrm{iles}$ daba tanta pena! Parecía que la estuvieran matando".

Tardó en hablar y en expresarse. No fue a la guardería y siempre ha estado muy poco con niños, no tiene primos. Con 3 años comenzó el colegio con mala 
adaptación, se volvió a hacer pis y lloraba mucho por su madre.

El padre ha estado trabajando fuera 2 años y la madre ha estado triste desde el primer año hasta los 4 años de Paloma. Dormía con ella, la malcriaba, todo lo que quería se lo daba, porque "no estaba su padre". La madre comenta que ella es muy miedosa, le da mucho miedo que se atraganten sus hijas: "¿Si les pasa algo por la noche y se ahoga...?".

La madre se pone a trabajar y Paloma vuelve a vomitar, no quiere ir al colegio y le dice que no quiere separarse de ella: "Me hace llorar, nunca la he dejado en ningún sitio, siempre he estado yo con ella, no la he dado libertad, no la he dejado hacer nada sin mí; yo no he salido con mi marido por estar siempre con la niña.... a lo mejor mis padres no han estado suficiente tiempo conmigo y yo he querido ser mejor que mis padres".

La madre cuenta que sus padres trabajaban mucho y que ella ha vivido con su abuela, desde los 5 a los 9 años. "Ahora cuando he sido madre, es cuando he echado de menos a mi madre".

\section{Alicia}

Alicia tiene 3 años, presenta una excesiva timidez que le impide relacionarse con los demás niños y le crea problemas de adaptación escolar. Su madre decidió dejar de trabajar para estar con su hija y no sabe si ha sido un error, pues están solas todo el día: "Yo soy su amiga y no quiere relacionarse con nadie". Aún duerme con sus padres.

Los padres describen que el embarazo fue deseado y bueno. No obstante, el parto acarreó complicaciones, fue una cesárea de urgencia, la intervinieron quirúrgicamente con 7 días de vida y estuvo 3 semanas en la UVI. La llegada a casa fue tensa porque la madre quería darla el pecho pero ya venía acostumbrada a los biberones, "no sé si me empeñé demasiado en darle el pecho...". Alicia dormía mal, la tenían que poner vertical.

Al año fue a la guardería, parece que se adaptó bien, pero como cogía muchas enfermedades, la sacaron, al padre "no le gustaba que llorara y vomitara". El padre comenta que lo han hecho todo por ella, han cambiado su vida completamente, no como otros padres. A los 2 años se cambiaron de casa y Alicia empezó a dormir con ellos, ahora la madre se tiene que acostar con ella para que se duerma. Se han obsesionado con hacerlo todo tan bien, preocupándose por todo lo de ella que creen que se han equivocado. Admiten que quizás la han sobreprotegido demasiado.

La madre de Alicia comenta que ella también era así, muy tímida, introvertida, 
la mejor de la clase, pero no le gustaba que la miraran ni hablar en público: "Mis padres me han sobreprotegido mucho, me han metido todos sus miedos y sigo siendo introvertida, poco sociable y sufro de insomnio". El padre también es muy introvertido, agobiante y acaparador, le cuesta hacer amigos, tuvo una depresión importante cuando se casó.

Alicia no sabe defenderse, necesita de algún adulto a su lado, llora por todo en el colegio y no disfruta con nada. La madre se da cuenta de que le está pasando lo mismo que le pasaba a ella de pequeña: "iTengo que cambiar yo!".

\section{Juan}

Juan tiene 7 años. Siempre ha sido difícil de llevar por sus nervios y su timidez. Tiene falta de atención, se evade, tiene la autoestima muy baja, se va a su mundo y no escucha. Cuando alguien está con él sí trabaja, pero si está solo, no hace nada.

Dormía mal desde el principio, tuvo cólicos del lactante y a los 2 meses una bronquiolitis. "Se ponía morado y les dijeron que tuvieran cuidado porque de un golpe de tos se podía asfixiar".

La madre reconoce que siempre lo tuvo encima, lo dormía en sus brazos, se aliviaba así, Ha dormido con ellos hasta los 3 años, cuando se quedó embarazada de su segundo hijo y les dio miedo que le diera patadas en la tripa. En una ocasión, con 8 meses, durmió toda la noche de un tirón y la madre se levantó para ver si respiraba. Explica que tenía mucho miedo a que le pasara algo, que se asfixiara y se pudiera morir.

La madre reconoce que ella ha estado siempre presente y que no le han fomentado la capacidad de esperar. Ella lo ha tenido en brazos haciendo las cosas de la casa, hasta los 2 años y medio, que ya no podía más por la tripa del nuevo embarazo. El padre dice que hay una relación muy especial entre Juan y su madre.

Con 12 meses, Juan va a la guardería, le dijeron que estaba muy "enmadrado", pero la cuidadora pudo hacerse con él. Habló pronto y claro, pero no jugaba y no se relacionaba, pegaba a la madre. $A$ los 3 años lo escolarizaron, le costó muchísimo adaptarse, ha tenido muchos problemas para relacionarse con los niños, no jugaba ni con sus primos.

Al padre le ha costado mucho interactuar con él, sentía que su hijo no le quería, que no conectaban. A lo largo de las entrevistas, el padre participa más con Juan y está menos rebelde, menos exigente, más sociable y se relaciona mejor.

\section{Carla}

Carla es una niña de 6 años, que ha dejado de comer en el colegio y desde 
hace 2 meses dice que no quiere ir al colegio. Parece que el profesor le estaba exigiendo demasiado y se había enfadado con ella, porque estaba bloqueada, no aprendía e iba a perder el curso. Desde que hablaron con el profesor, se ha normalizado la situación.

El embarazo y parto de Carla fueron normales, y recibió lactancia materna hasta los 2 años y medio. A los 2 años fue a la guardería, pero no se adaptaba, y se pasó el año entero pegada a su mochila, sin jugar con los otros niños. Cuando estaba en la piscina pública, con 2 años de edad, iba corriendo donde estaba su madre, y esta sacaba el pecho y Carla se ponía a chupar, no podía dejarla llorar.

La madre duerme con Carla y ahora también con el hermano pequeño, dice que "a ella no le importa" y al padre tampoco le importa que ella duerma con los niños. A él le gusta dormir solo, cuando se casaron quería una cama individual, así que a raíz de tener a la hija él vio el cielo abierto para dormir solo.

La madre empieza a hablar de que "se siente invadida, que el hijo se quiere despegar pero que ella no le deja y que con Carla es una lucha entre las dos". Se da cuenta que de pequeña no la ha sacado al parque, se iba a casa de los abuelos maternos a comer y allí se quedaba toda la tarde. Ella se volcó mucho en Carla, hasta su madre le decía que era demasiado protectora.

La madre de Carla le dice a su marido que van a tener que dormir juntos y él contesta que si ahora después de 6 años en esta situación quiere dormir con él, se niega rotundamente. Ella comenta en la consulta dirigiéndose a su esposo: "Con los niños no puedo y tú no quieres, y ahora ¿qué hago yo?".

\section{Intervención}

A partir de lo visto hasta ahora queda claro que la persistencia anómala de la ansiedad de separación es un signo de inmadurez psicológica y que las pautas de intervención van orientadas a fomentar el sentimiento de seguridad y su capacidad de autonomía que, como base de su personalidad, el niño está consolidando. Para ello es imprescindible un fuerte y constante apoyo por parte de los padres, combinado con estimulación de la independencia del niño y respeto a la misma, en lugar de minar la confianza en sí mismo, proporcionar las condiciones en la cual el niño pueda desarrollarse mejor. Los escenarios en los que se puede intervenir en esta dirección no son otros que aquellos en los que en el día a día el niño debe progresar desde la dependencia absoluta de sus padres hasta una au- 
tonomía personal, como son el ser capaz de dormir solo, alcanzar la alimentación racional, o la exploración del entorno y regulación de sus miedos.

\section{Dormir}

En relación con los problemas para dormir, los más comunes son la tardanza para conciliar el sueño y la reacción de angustia y llanto ante la percepción de la ausencia de los padres. Desde esta perspectiva, la asignación de una habitación aparte a los niños para que duerman separados de sus padres es un elemento desde el que se puede promover la aparición de la autonomía psicológica que el niño necesita.

Para propiciar este aprendizaje es imprescindible, en primer lugar, acostumbrar a los bebés a un horario y a la regularidad de los acontecimientos que rodean al momento de dormir: baño relajante, cena, lavado de dientes, lectura de cuento, peluche, beso y buenas noches, apagar la luz y cerrar la puerta. Sin la menor duda es preferible apagar la luz. Al hacerlo parece ser que se activa la secreción de melanotonina, que favorece la normal regulación de las fases del sueño y facilita el buen descanso.

Hay que recordar que el método de despedida que se utilice en esa ocasión será el que tenderán a reclamar para vol- ver a dormirse en los despertares que ocasionalmente pueden aparecer a lo largo de la noche. Ante ello, lo mejor es acudir a atenderles con absoluta parquedad, asegurándose que no les ocurre nada malo y cuidando en extremo no otorgarles gratificaciones suplementarias (caricias, besos o frases cariñosas, etc.).

La continencia expresiva del adulto (tanto de amor como de enfado) ante las demandas nocturnas o el despertar infantil, es fundamental. Siempre se ha de dar por buena la despedida realizada al acostarles la primera vez. De afrontar una insistencia infantil muy manifiesta, no queda otro remedio que disminuir progresivamente la premura en responder. Y en el caso de que el método de llamada sean los lloros y los gritos es necesario soportarlos hasta que se agoten sin caer en la pérdida de nervios. De ceder se entra en una difícil escalada en la que el incremento de la insistencia y del volumen no parece tener fin.

\section{Comer}

La alimentación es otro de los escenarios fundamentales donde el niño debe conquistar su autonomía desde la dependencia absoluta de lactancia materna hasta la alimentación racional e independiente. Para fomentar este salto hacia su autonomía es necesario confiar absoluta- 
mente en el apetito de los niños, considerándolo el mejor indicador de su mayor o menor necesidad de alimentarse.

Esta fuente de placer tan completa que supone la alimentación infantil durante los primeros meses de vida del niño no debe modificarse, pero lo que sí que poco a poco hay que cambiar es la absoluta pasividad infantil inicial y la total dedicación materna mientras el hijo se alimenta. Durante los primeros 3 ó 4 años de vida el niño debe aprender a comer solo, tomando conciencia plena de su hambre como expresión de una de sus necesidades naturales (el comer por necesidad propia y no de la madre, del padre, de los abuelos o de quien corresponda). Aprender a comer sin ayuda contribuye enormemente a la superación de la ansiedad de separación, la génesis del sentimiento de autonomía y, probablemente, a la paralela consolidación de una identidad menos fusional y dependiente.

Lo aconsejable es reducir paulatinamente la atención alrededor de la necesidad de que coman, centrándola especialmente en la enseñanza de su manipulación de los cubiertos. A su vez, complementariamente, se incrementará la actividad comunicativa al margen de las comidas, pero, sobre todo, hay que procurar no dar a los niños más atención cuando comen mal que cuando lo hacen bien.
Cuando los niños son muy lentos o malos comedores es preferible dejarles en una mesa solos y darles un tiempo determinado para cada plato (10 ó 15 minutos). Y si continúan sin comer, es mejor retirarles la comida con la seguridad de que no pasarán muchas horas sin sentir hambre verdadera (cuidando que no piquen entre comidas o coman golosinas). Lo importante es no obsesionarse con las cantidades ingeridas ni por cuántas veces se quedan sin comer.

Otra cuestión fundamental es que cuando los niños son capaces de llevarse la cuchara a o el tenedor a la boca por sí mismos, hay que fomentar esta maniobra, así, aunque se les ayude a partir los alimentos y a pincharlos con el tenedor, no se debe llevar el cubierto con el alimento hasta su boca, pues al hacerlo el adulto se entremete en el esquema corporal del niño. El llevarles la comida a la boca es lo más parecido a trasladarles a un paraíso alejado de la realidad de este mundo, en el que las satisfacciones materiales se logran sin coste alguno.

\section{Miedos}

En el trasfondo de muchos de los miedos infantiles, la angustia que verdaderamente subyace y se manifiesta es la ansiedad de separación. En este punto, es necesario subrayar que más importante 
aún que cómo regule el niño sus miedos, está el hecho de cómo los manejan sus padres. No se puede esperar que el niño pueda llegar a dominar su miedo cuando surge una persona (generalmente uno de los padres o ambos) que llega a tener más miedo a la reacción del pequeño que el propio niño al objeto de su temor, desviviéndose por ir por delante (aunque emocionalmente sea ir por detrás) para retirar del camino cualquier mínima causa de los miedos.

El adulto ha de perder el miedo que indirectamente también le desencadena el objeto o la situación que atemoriza a su pequeño (por resultarle insoportable la reacción que este pueda llegar a tener). Si el adulto no supera las reacciones infantiles de pánico, se descalifica para pretender que los niños consigan superar su miedo. Cuando la madre o el padre o los abuelos, o todos ellos, tiemblan al pensar en el espanto que tanto le asusta (por ejemplo, la separación) y se lo evitan, están impidiendo el desarrollo de la autonomía y madurez del niño.

Por lo tanto, la actitud recomendable se basa en mantener cierto respeto hacia el miedo del niño sin compartirlo, demostrando principalmente la ausencia total de sintonía en lo que respecta a la preocupación y la ansiedad, expresándole una crítica ponderada. Asimismo, también resulta conveniente ir incrementando progresivamente la presión que ha de ejercerse para que los niños se enfrente por sí mismos a la fuente del miedo. Es decir, se puede dejar a los niños poco a poco y con prudencia ante las circunstancias que los atemorizan.

\section{Conclusiones}

El objetivo de las intervenciones terapéuticas es trabajar fundamentalmente con los padres para contener sus ansiedades, analizar las dificultades que tienen con sus hijos, que suelen estar relacionadas con sus propios miedos a la separación y a la pérdida. Asimismo, es necesario incrementar la capacidad de comprensión y de seguridad de ellos mismos como padres, despejando la relación con sus hijos de sus propios fantasmas del pasado que estaban interfiriendo en sus actuaciones.

Es fundamental la detección precoz de estos padres inseguros, pues cuanto antes intervengamos facilitándoles su función de cuidador seguro y disponible vamos a evitar comportamientos alterados en los niños, en sus procesos regulatorios, funcionales y madurativos. 


\section{Bibliografía}

1. Gracia Clavero F. La nueva educación. Zaragoza: Mira Editores; 2002.

2. Jalenques, I, Lachal C, Coudert AJ. Los cuadros de ansiedad en el niño. Barcelona: Masson; 1994.

3. Cassidy J, Shaver PR. Handbook of attachment: theory, research and clinical applications. New York: Guilford; 1999.

4. Winnicott DW. El proceso de maduración en el niño. Estudios para una teoría del desarrollo emocional. Barcelona: Laia; 1981.

5. Méndez FX, Olivares J, Bermejo RM. Características clínicas y tratamiento de los miedos, fobias y ansiedades específicas. En: Caballo V, Simón MA (eds.). Manual de Psicología clínica infantil y del adolescente. Madrid: Pirámide; 2001. p. 87-124.
6. Bragado MC. Terapia de conducta en la infancia: trastornos de ansiedad. Madrid: Fundación Universidad-Empresa; 1994.

7. Sandín B. Ansiedad, miedos y fobias en niños y adolescentes. Madrid: Dykinson; 1997.

8. Berrueco Arjona J. Las consecuencias de la educación a demanda. Rev Pediatr Aten Primaria. 2007:9:291-9.

9. Fonagy P. Teoría del apego y psicoanálisis. Barcelona: Espaxs; 2004.

10. Bowlby J. Vínculos afectivos: formación, desarrollo y pérdida. Madrid: Morata; 1986.

11. Main M, Solomon J. Discovery of a new insecure-disorganized/disoriented attachment pattern. In: Blazelton T, Yogman M (eds.). In support of families. New York: Ablex; 1986. p. 213-39. 